\title{
Disease susceptibility genes shared by primary biliary cirrhosis and Crohn's disease in the Japanese population
}

\author{
Yoshihiro Aiba ${ }^{1}$, Keiko Yamazaki ${ }^{2}$, Nao Nishida ${ }^{3,4}$, Minae Kawashima ${ }^{3,5}$, Yuki Hitomi ${ }^{3}$, Hitomi Nakamura ${ }^{1}$, \\ Atsumasa Komori ${ }^{1,6}$, Yuta Fuyuno ${ }^{2,7}$, Atsushi Takahashi ${ }^{8}$, Takaaki Kawaguchi ${ }^{9}$, Masakazu Takazoe ${ }^{9}$, \\ Yasuo Suzuki ${ }^{10}$, Satoshi Motoya ${ }^{11}$, Toshiyuki Matsui ${ }^{2,12}$, Motohiro Esaki ${ }^{7}$, Takayuki Matsumoto ${ }^{13}$, \\ Michiaki Kubo $^{2}$, Katsushi Tokunaga ${ }^{3}$ and Minoru Nakamura ${ }^{1,6,14}$
}

We previously identified TNFSF15 as the most significant susceptibility gene at non-HLA loci for both primary biliary cirrhosis (PBC) and Crohn's diseases (CD) in the Japanese population. The aim of this study is to identify further disease susceptibility genes shared by PBC and CD. We selected 15 and 33 genetic variants that were significantly associated with PBC and CD, respectively, based on previously reported genome-wide association studies of the Japanese population. Next, an association study was independently performed for these genetic variants in CD (1312 CD patients and 3331 healthy controls) and PBC (1279 PBC patients and 1015 healthy controls) cohorts. Two CD susceptibility genes, ICOSLG rs2838519 and IL12B rs6556412, were also nominally associated with susceptibility to PBC $\left(P=3.85 \times 10^{-2}\right.$ and $P=8.40 \times 10^{-3}$, respectively). Three PBC susceptibility genes, CXCR5 rs6421571, STAT4 rs7574865 and NFKB1 rs230534, were nominally associated with susceptibility to $C D\left(P=2.82 \times 10^{-2}, P=3.88 \times 10^{-2}\right.$ and $P=2.04 \times 10^{-2}$, respectively). The effect of ICOSLG and CXCR5 variants were concordant but the effect of STAT4, NFKB1 and IL12B variants were discordant for PBC and CD. TNFSF15 and ICOSLG-CXCR5 might constitute a shared pathogenic pathway in the development of PBC and CD in the Japanese population, whereas IL12B-STAT4-NFKB1 might constitute an opposite pathogenic pathway, reflecting the different balance between Th1 and Th17 in the two diseases.

Journal of Human Genetics (2015) 60, 525-531; doi:10.1038/jhg.2015.59; published online 18 June 2015

\section{INTRODUCTION}

Primary biliary cirrhosis (PBC) is an autoimmune liver disease characterized by the destruction of intrahepatic small bile ducts with portal inflammation, also known as chronic nonsuppurative destructive cholangitis. It results in liver cirrhosis and hepatic failure over several decades. Although immune-mediated damage of intrahepatic biliary epithelial cells and hepatocytes is considered part of the pathogenesis of $\mathrm{PBC}$, the details of the involved pathogenic mechanisms remain unknown. Recently, genome-wide association studies (GWAS) and Immunochip analyses of PBC have identified a total of 28 susceptibility loci for $\mathrm{PBC}$ in Caucasian populations. ${ }^{1-3}$ In addition, two novel PBC susceptibility genes, TNFSF15 and POU2AF1, which were not identified in Caucasian populations, were identified by GWAS in the Japanese population. In addition, a total of 10 Caucasian PBC susceptibility loci, HLA, DENND1B, STAT4, CD80, NFKB1, IL7R, CXCR5, TNFAIP2, 17q21 and MAP3K7IP1, were replicated in the Japanese population. ${ }^{4}$ These genes are implicated in immune responses including innate and adaptive immune responses, indicating that innate and adaptive immune signaling pathways have an important role in the pathogenesis of PBC.

Crohn's disease (CD) is an inflammatory bowel disease (IBD) characterized by remitting and relapsing inflammation of the intestinal tract. Epidemiological studies show genetic and environmental factors are involved in its pathogenesis. As for genetic factors, a total of 140

${ }^{1}$ Clinical Research Center, National Hospital Organization, Nagasaki Medical Center, Omura, Japan; ${ }^{2}$ Laboratory for Genotyping Development, Center for Integrative Medical Science, Institute of Physical and Chemical Research (RIKEN), Yokohama, Japan; ${ }^{3}$ Department of Human Genetics, Graduate School of Medicine, The University of Tokyo, Tokyo, Japan; ${ }^{4}$ The Research Center for Hepatitis and Immunology, National Center for Global Health and Medicine, Chiba, Japan; ${ }^{5}$ Japan Science and Technology Agency (JST), Tokyo, Japan; ${ }^{6}$ Department of Hepatology, Nagasaki University Graduate School of Biomedical Sciences, Nagasaki, Japan; ${ }^{7}$ Department of Medicine and Clinical Science, Graduate School of Medical Sciences, Kyushu University, Fukuoka, Japan; ${ }^{8}$ Laboratory for Statistical Analysis, Center for Integrative Medical Sciences, RIKEN, Tokyo, Japan; ${ }^{9}$ Department of Medicine, Division of Gastroenterology, Tokyo Yamate Medical Center, Tokyo, Japan; ${ }^{10}$ Department of Internal Medicine, Faculty of Medicine, Toho University, Chiba, Japan; ${ }^{11}$ Department of Gastroenterology, Sapporo Kosei Hospital, Sapporo, Japan; ${ }^{12}$ Department of Gastroenterology, Fukuoka University Chikushi Hospital, Fukuoka, Japan; ${ }^{13}$ Division of Gastroenterology, Department of Internal Medicine, Iwate Medical University, Iwate, Japan and ${ }^{14}$ Headquarters of PBC Research in the National Hospital Organization Study Group for Liver Disease in Japan (NHOSLJ) and gp210 working in Research Program of Intractable Hepatoboliary Disease Study Group supported by the Ministry of Health, Labour, and Welfare of Japan, Clinical Research Center, National Hospital Organization Nagasaki Medical Center, Nagasaki, Japan

Correspondence: Dr M Nakamura, Clinical Research Center, National Hospital Organization, Nagasaki Medical Center, 2-1001-1 Kubara, Omura, 856-8562, Japan. E-mail: nakamuram@nagasaki-mc.com

Received 10 February 2015; revised 1 May 2015; accepted 1 May 2015; published online 18 June 2015 
susceptibility loci have been identified by GWAS and a subsequent meta-analysis in Caucasian populations. ${ }^{5}$ In the Japanese population, TNFSF15, STAT3, ELF1, JAK2, RUNX3, C1orf94, TBC1D1, CCDC6, $6 \mathrm{p} 21$ and 2p25 loci were identified as susceptibility genes by GWAS, and 27 out of 71 Caucasian CD susceptibility loci were associated with CD in Japanese replication studies. ${ }^{6-8}$ The CD susceptibility genes TNFSF15, STAT3, IL12B, CCR6, which were identified in both Caucasian and Japanese populations, are genes associated with Th17, indicating the importance of the Th17 signaling pathway in the pathogenesis of CD.

In contrast to Caucasian populations, TNFSF15 was found to be the most significant disease susceptibility gene in both $\mathrm{PBC}$ and $\mathrm{CD}$ in the Japanese population. ${ }^{4,7}$ Since TL1A encoded by TNFSF15 is involved in apoptosis and immune responses leading to Th1 and Th17 differentiation, it is likely that TL1A has an important role in the development of both diseases through these mechanisms. ${ }^{9}$ Although the age of onset and gender distribution of these two diseases are different and the concomitance of $\mathrm{PBC}$ and $\mathrm{CD}$ is very rare, these two diseases have several shared clinical characteristics such as epithelial cell destruction with inflammation and granuloma formation at locations involved in the enterohepatic circulation of bile salts. In addition, many disease susceptibility genes are shared among various autoimmune diseases, indicating the presence of shared pathogenic pathways among autoimmune disease. ${ }^{10}$ Previous studies have shown the associations of HLA region, in particular HLA-DRB1, with susceptibility to PBC or CD in the Japanese population. DRB $1{ }^{\star} 0803$ and $\mathrm{DRB} 1^{\star} 0405$ alleles confer susceptibility to $\mathrm{PBC}$ development, whereas $\mathrm{DRB}^{\star} 1101, \mathrm{DRB} 1^{\star} 1302$ and $\mathrm{DRB}^{\star} 1501$ alleles protect against the disease development. ${ }^{1,12} \mathrm{DRB} 1^{\star} 0405$ allele confers susceptibility to $\mathrm{CD}$ development, whereas $\mathrm{DRB} 1^{\star} 1502$ allele protects against the development. ${ }^{13,14}$ Thus, DRB1 ${ }^{\star} 0405$ is a shared susceptibility allele for PBC and CD in the Japanese population. In the present study, therefore, to further identify shared susceptibility genes at nonHLA loci and pathogenic pathways for PBC and CD in the Japanese population, we performed a comparative case-control association study of CD susceptibility genes with $\mathrm{PBC}$ and vice versa.

\section{MATERIALS AND METHODS}

\section{Patients and controls}

A total of 1279 PBC patients ( $88.6 \%$ female; median age 58 years, range $23-89$ years) and 1015 healthy controls ( $58.7 \%$ female, median age 36 years, range 24 87 years) were recruited by the National Hospital Organization Study Group for Liver Disease in Japan. These PBC patients and controls were 99\% and 96\% overlapped with previous study ${ }^{4}$, respectively. In addition, $1312 \mathrm{CD}$ patients (30.0\% female; median age 22 years, range 6-79 years, 99\% overlapping with previous study $^{6}$ ) and 3331 healthy controls (44.5\% female; median age unknown, range 3-96 years, 98\% overlapping with previous GWAS study ${ }^{7}$ ) were recruited at the RIKEN Yokohama Institute. All CD patients and PBC patients were diagnosed according to previously described diagnostic criteria. ${ }^{4,7}$ PBC patients who had acute hepatitis, chronic hepatitis B or C infection, alcoholic liver disease or other chronic liver diseases were excluded from this study. Informed consent was obtained from all cases and controls before participation in this study. This study was approved by the ethics committees of National Hospital Organization Study Group for Liver Disease in Japan and RIKEN Yokohama Institute.

\section{DNA preparation}

Genomic DNA was extracted from the peripheral whole blood of subjects using NucleoSpin Blood Quick Pure (Macherey-Nagel, Düren, Germany).

\section{SNP selection and genotyping}

We reviewed the literature for GWAS and replication studies related to susceptibility genes to $\mathrm{PBC}$ or $\mathrm{CD}$ in the Japanese population. ${ }^{4,6,7} \mathrm{We}$ selected genetic polymorphisms located at non-HLA loci that were significantly associated with $\mathrm{PBC}$ or $\mathrm{CD}$ in GWAS $\left(P<1 \times 10^{-4}\right)$ and replication studies $\left(P<5 \times 10^{-2}\right)$. When there were several candidate genetic polymorphisms at a putative susceptibility locus, the genetic polymorphism with the lowest $P$-value was selected for the present comparative association study. Selected genetic polymorphisms were genotyped using the Taq Man assay (Applied Biosystems, Foster City, CA, USA), DigiTag2 assay ${ }^{15}$, or multiplex polymerase chain (PCR)-based Invader assay (Third Wave Technologies, Madison, WI, USA). ${ }^{16}$

\section{Statistical analysis}

Hardy-Weinberg equilibrium was evaluated with the $\chi^{2}$ goodness-of-fit test. The frequencies of the alleles for each genetic polymorphism were compared between cases and controls using the $\chi^{2}$-test. We considered $P<0.05$ to indicate a nominal association. Multiple testing in the allele test was corrected by using Bonferroni's method. We considered an association to be significant when the $P$-value was $<0.05$ even after multiple comparisons.

\section{RESULTS}

\section{Selection of genetic polymorphisms}

To identify susceptibility genes shared by PBC and CD, we selected a total of 15 and 33 genetic polymorphisms associated with $\mathrm{PBC}$ and $\mathrm{CD}$, respectively. Of the susceptibility loci for CD, TNFSF15 and STAT3 had two genetic polymorphisms associated with CD in previous studies (rs6478106 and 11871801 at TNFSF15 and rs9891119 and rs3810936 at STAT3). ${ }^{6,7}$ Rs6478106 at TNFSF15 and rs9891119 at STAT3 were selected for the present association studies because these genetic polymorphisms had lower $P$-values than other genetic polymorphisms, respectively, (rs11871801 at TNFSF15 and rs3810936 at STAT3) in the previous CD association studies.

\section{Shared genetic polymorphisms that confer susceptibility to both $\mathrm{PBC}$ and $\mathrm{CD}$ in the Japanese population}

Of 15 PBC susceptibility loci, four loci were associated with $\mathrm{CD}$ : TNFSF15 (rs4979462) was significantly associated with CD and STAT4 (rs7574865), NFKB1 (rs230534) and CXCR5 (rs6421571) were nominally associated with $\mathrm{CD}$ (Table 1). Risk alleles for TNFSF15 (rs4979462) and CXCR5 (rs6421571) with respect to $\mathrm{CD}$ were the same as those for $\mathrm{PBC}$. On the other hand, the risk alleles at the STAT4 (rs7574865) and NFKB1 (rs230534) loci for CD were the opposite of those for PBC. Similarly, we selected 33 susceptibility genes associated with $\mathrm{CD}$ and examined the association between these genetic polymorphisms and PBC. TNFSF15 (rs6478106) and two other loci, ICOSLG (rs2838519) and IL12B (rs6556412), were significantly and nominally associated with susceptibility to $\mathrm{PBC}$, respectively (Table 2). The risk alleles of TNFSF15 (rs6478106) and ICOSLG (rs2838519) for $\mathrm{PBC}$ were the same as those for $\mathrm{CD}$, whereas the risk allele of IL12B (rs6556412) for PBC was opposite to that for CD. Susceptibility genes shared by $\mathrm{PBC}$ and $\mathrm{CD}$ in the Japanese population are illustrated in Figure 1.

\section{DISCUSSION}

In this study, 4 out of $33 \mathrm{CD}$ susceptibility loci and 3 out of $15 \mathrm{PBC}$ susceptibility loci showed associations with $\mathrm{PBC}$ and $\mathrm{CD}$, respectively. Thus, we identified TNFSF15, IL12B, ICOSLG, CXCR5, STAT4 and NFKB1 as shared susceptibility genes for these two diseases in the Japanese population, although the association of five loci (IL12B, ICOSLG, CXCR5, STAT4 and NFKB1) except for 
:

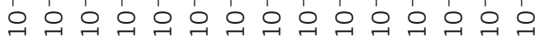

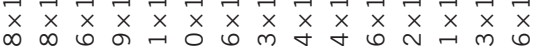

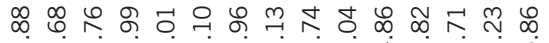

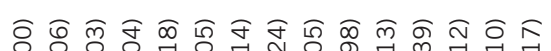

(ำ

$\approx$

ฟ

द o

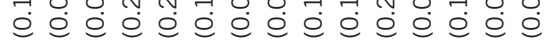

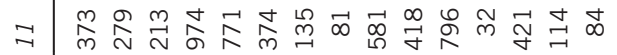

这

$\approx$

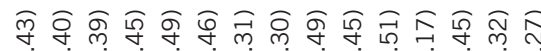

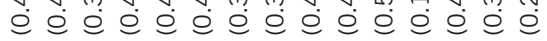

$\approx$

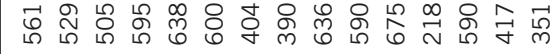

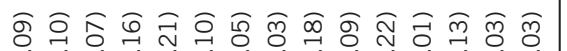

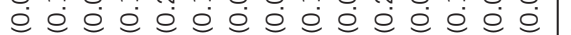

$\approx$

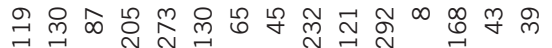

旁

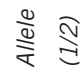

$100 \vdash 0<000<00 \ll<1$

.

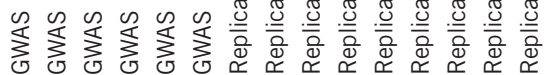

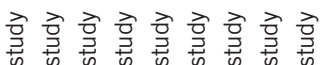

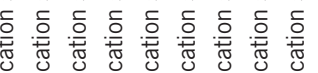

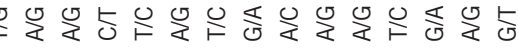

离

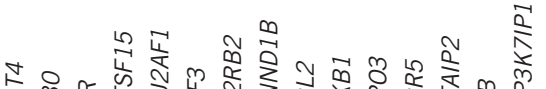

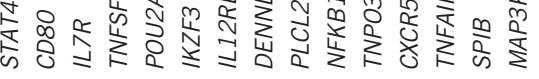

m్ల

离

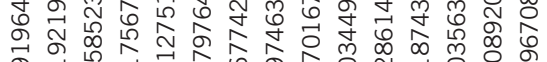

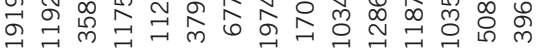

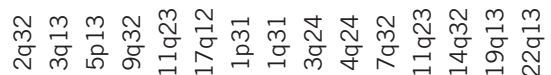

s

$\sum_{\text {के }}^{\frac{0}{5}}$

운

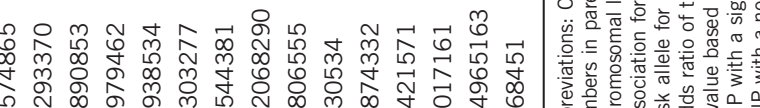

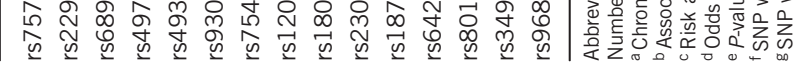


$\stackrel{10}{\circ}$

บิ

ก๊

8

迹

ฟ

$\approx$$$
\exists
$$

迹

N

$\frac{0}{5}$ के

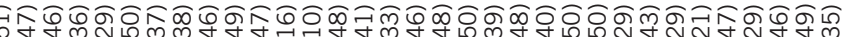

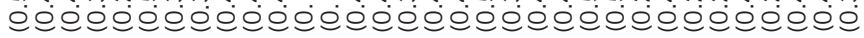

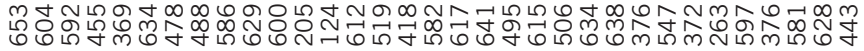
굿⿵冂卄

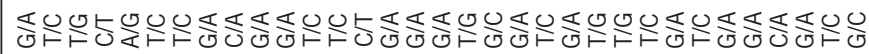

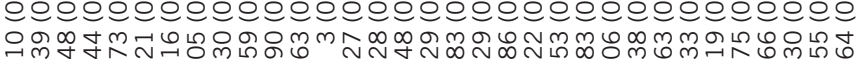
mm $m$ 守

(5)

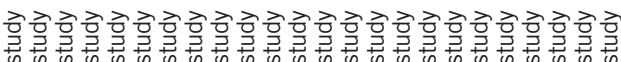

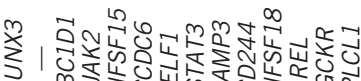
은.

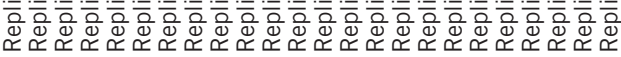

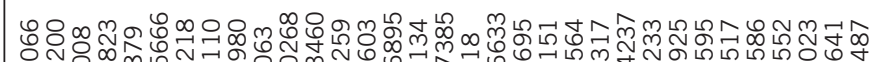
m m N mํำ

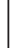

(1)

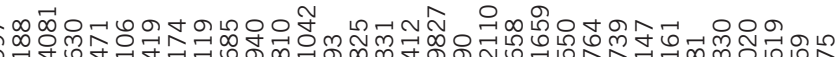

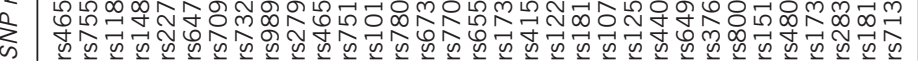

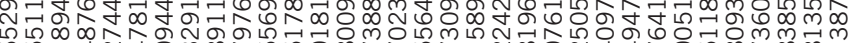
设

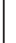


TNFSF15 is not convincing but suggestive. Among these newly identified shared susceptibility loci, four loci, TNFSF15 (rs4979462 and rs6478106), CXCR5 (rs6421571) and ICOSLG (rs2838519), shared the same risk alleles but for three other loci, STAT4 (rs7574865), NFKB1 (rs230534) and IL12B (rs6556412), the risk alleles were different for the two diseases, suggesting that the shared pathogenic pathways may operate in the same or opposite direction in $\mathrm{PBC}$ and CD.

Among the shared susceptibility loci, the odds ratio at the TNFSF15 locus (rs6478106, odds ratio $1.46, P=2.05 \times 10^{-10}$ in $\mathrm{PBC}$; and rs4979462, odds ratio $1.96, P=1.68 \times 10^{-37}$ in $C D$ ) was higher than that of other non-HLA loci, and serum and local expression levels of TNFSF15 are increased in both PBC and CD patients, ${ }^{17-19}$ indicating

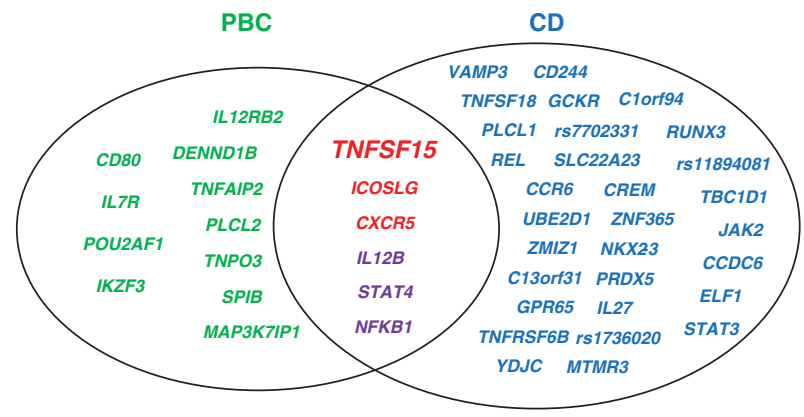

Figure 1 Comparison of susceptibility genes between PBC and CD in the Japanese population. Shared susceptibility genes between PBC and CD in the same direction (red) or in the opposite direction (purple). CD, Crohn's disease; PBC, primary biliary cirrhosis. that TNFSF15 has an important role in the development of both diseases. A previous study showed that the risk allele of TNFSF15 rs6478106, which is located in the $5^{\prime}$-flanking region, enhances the transcriptional efficiency of TNFSF $15 .{ }^{20}$ In addition, we recently found that the risk allele of TNFSF15 rs4979462, which is located in intron 1, enhances the transcription efficiency of TNFSF15 via the formation of the transcriptional factor NF-1 binding site. ${ }^{21}$ The two TNFSF15 genetic polymorphisms, rs6478106 and rs4979462, showed strong but not complete linkage disequilibrium in the $\mathrm{PBC}\left(r^{2}=0.64\right.$, $\left.D^{\prime}=0.82\right)$ and CD cohorts $\left(r^{2}=0.80, D^{\prime}=0.90\right)$ in the present study, suggesting that these TNFSF15 genetic polymorphisms cooperatively regulate the expression level of TNFSF15 and confer susceptibility to both diseases.

Although the functional significance of CXCR5 rs6421571 remains unclear, the risk allele for IBD associated with ICOSLG rs7282490 was recently reported to downregulate ICOSLG expression and signaling of NOD2-induced ICOS (a receptor for ICOSLG) in monocyte-derived dendritic cells, leading to impairment of innate immune responses to the microbe. ${ }^{22}$ In the HapMap database, ICOSLG rs7282490 shows strong linkage disequilibrium with the ICOSLG rs2838519 genetic polymorphism, which is associated with both PBC and CD susceptibility in the present study. These reports indicate that loss of function in ICOSLG rs7282490 might be associated with a shared clinical feature, granuloma formation, in both diseases. Both ICOS and CXCR5 are characteristic cell surface markers on follicular helper T-cells that are mainly present in the germinal center. They are implicated in the differentiation and maturation of B cells. ${ }^{23}$ It has been reported that follicular helper T-cells participate in the pathogenesis of several

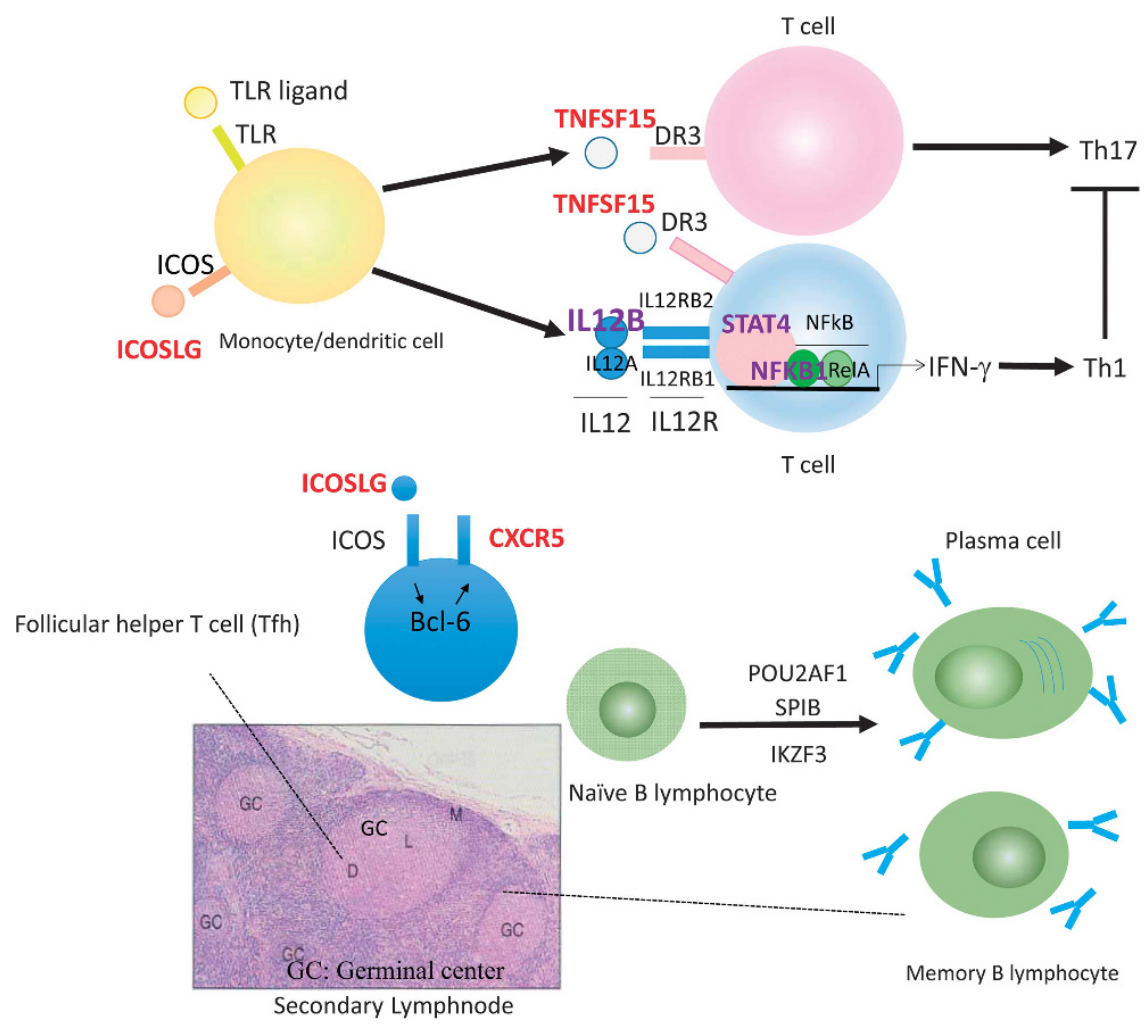

Figure 2 The role of suggestive shared susceptibility genes for PBC and CD in innate and adaptive immune responses. The shared susceptibility genes for PBC and CD are in same direction (red) or in the opposite direction (purple). Abbreviations: CD, Crohn's disease; PBC: primary biliary cirrhosis. 
autoimmune diseases including systemic lupus erythematosus and rheumatoid arthritis. ${ }^{24}$ The number of follicular helper T-cells in the peripheral blood or spleen is higher in both PBC and $\mathrm{CD}$, and is correlated with disease activity, including autoantibody production, cytokine production and response to ursodeoxycholic acid treatment in PBC. ${ }^{25,26}$ Collectively, these results indicate that ICOSLG and CXCR5 genetic polymorphisms may play important roles in both innate and adaptive immunity in these two diseases.

In the present study, three genetic polymorphisms involved in the IL12 signaling pathway, IL12B rs6556412, STAT4 rs7574865 and NFKB1 rs230534, showed an association with susceptibility to PBC and $\mathrm{CD}$. Although the functional significance of these genetic polymorphisms remains unclear, these risk alleles are in opposite directions in these two diseases. IL12 is a major cytokine associated with the development of Th1 responses, and as a major Th1 cytokine, IFN- $\gamma$ suppresses Th17 differentiation and development. ${ }^{27}$ These data indicate the possibility that differential IL12 signaling might affect the relative contribution of Th1/Th17 immune responses in the pathogenesis of both diseases.

In conclusion, we identified five shared susceptibility genes, CXCR5, ICOSLG, STAT4, IL12B and NFKB1, in addition to TNFSF15, which $\mathrm{PBC}$ and $\mathrm{CD}$ have in common in the Japanese population. In particular, risk alleles for TNFSF15, CXCR5 and ICOSLG have the same effects on the susceptibility to these two diseases, suggesting that these molecules might constitute a common pathogenic pathway in the development of $\mathrm{PBC}$ and $\mathrm{CD}$. On the other hand, risk alleles for IL12B, STAT4 and NFKB1 are opposite for these two diseases, suggesting that the regulation of Th1 and Th17 polarization via the IL12-STAT4-NFKB signaling pathway might be in the opposite direction for these two diseases (Figure 2). ${ }^{28}$ These results might help to clarify the pathogenesis of $\mathrm{PBC}$ and $\mathrm{CD}$. The functional significance of the shared genetic polymorphisms still remains largely unknown, further analysis is required to elucidate the significance of the genetic polymorphisms identified in the present study.

\section{CONFLICT OF INTEREST}

The authors declare no conflict of interest.

\section{ACKNOWLEDGEMENTS}

We thank the PBC patients and healthy volunteers for participating in this study, as well as the following members for obtaining informed consent and collecting serum and DNA samples from PBC patients: PBC Study Group of NHSOLJ; Drs. Hiromi Ishibashi, Hiroshi Yatsuhashi, Seigo Abiru, Shinya Nagaoka (NHO Nagasaki Medical Center), Makoto Nakamuta, Motoyuki Kohjima (NHO Kyushu Medical Center), Hajime Ota (NHO Kanazawa Medical Center), Tatsuji Komatsu (NHO Yokohama Medical Center), Toshiki Nikami, Jinya Ishida, Takeo Saoshiro (NHO Nishisaitama Hospital), Hirotsugu Kouno, Hirotaka Kouno (NHO Kure Medical Center), Hiroshi Kamitsukasa (NHO Tokyo Hospital), Kiyoshi Furuta (NHO Matsumoto Medical Center), Toyokichi Muro, Tsutomu Yamashita (NHO Oita Medical Center), Naohiko Masaki (National Center for Global Health and Medicine), Yusuke Shimada (NHO National Disaster Medical Center), Yukio Watanabe, Yoko Nakamura (NHO Sagamihara Hospital), Masaaki Shimada, Noboru Hirashima (NHO Nagoya Medical Center), Toshiki Komeda (NHO Kyoto Medical Center), Kazuhiro Sugi (NHO Kumamoto Medical Center), Eiichi Takesaki, Yuko Nagaoki (NHO Higashi-Hiroshima Medical Center), Yukio Ohara (NHO Hokkaido Medical Center), Hiroshi Mano (NHO Sendai Medical Center), Haruhiro Yamashita, Kouki Matsushita (NHO Okayama Medical Center), Keisuke Ario (NHO Ureshino Medical Center), Masahiko Takahashi (NHO Tokyo Medical Center), Tetsuo Yamamoto (NHO Yonago Medical Center),
Fujio Makita (NHO Nishigunma Hospital), Hideo Nishimura (NHO Asahikawa Medical Center), Hitoshi Takagi, Atsushi Naganuma (NHO Takasaki General Medical Center) and Kaname Yoshizawa (NHO Shinshu Ueda Medical Center), Eiji Mita (NHO Osaka Medical Center); gp210 working in Research Program of Intractable Hepatoboliary Disease Study Group supported by the Ministry of Health, Labour and Welfare of Japan: Drs. Atsushi Tanaka, Hajime Takikawa (Department of Medicine, Teikyo University School of Medicine, Tokyo, Japan), Mikio Zeniya (Department of Gastroenterology and Hepatology, Tokyo Jikei University School of Medicine, Tokyo, Japan), Etsuko Hashimoto, Makiko Taniai (Department of Medicine and Gastroenterology, Tokyo Women's Medical Universiy, Tokyo, Japan), Hiromasa Ohira (Department of Gastroenterology and Rheumatic Diseases, Fukushima Medical University of Medicine, Fukushima, Japan), Kazuhide Yamamoto, Yasuhiro Miyake (Department of Gastroenterology and Hepatology, Okayama University Graduate School of Medicine, Dentistry and Pharmaceutical Sciences, Okayama, Japan), Morikazu Onji, Masanori Abe (Department of Gastroenterology and Metabology, Ehime University Graduate School of Medicine, Matsuyama, Japan), Shuichi Kaneko, Masao Honda, Kuniaki Arai (Department of Gastroenterology, Kanazawa University Graduate School of Medicine, Kanazawa, Japan), Satoshi Yamagiwa (Division of Gastroenterology and Hepatology, Niigata University Graduate School of Medical and Dental Sciences, Niigata, Japan), Kazuhiko Nakao, Hidetaka Shibata (Department of Gastroenterology and Hepatology, Nagasaki University Graduate School of Biomedical Sciences, Nagaski, Japan), Takafumi Ichida (Department of Gastroenterology and Hepatology, Juntendo University Shizuoka Hospital, Shizuoka, Japan), Masataka Seike (First Department of Internal Medicine, Faculty of Medicine, Oita University, Oita, Japan), Takeji Umemura, Satoru Joshita (Division of Gastroenterology and Hepatology, Department of Medicine, Shinshu University School of Medicine, Matsumoto, Japan), Yoshiyuki Ueno (Department of Gastroenterology,Yamagata University Faculty of Medicine,Yamagata, Japan), Shotaro Sakisaka, Yasuaki Takeyama (Department of Gastroenterology and Medicine, Fukuoka University School of Medicine, Fukuoka, Japan), Shinji Shimoda (Department of Medicine and Biosystemic Science, Kyushu University Graduate School of Medical Sciences, Fukuoka, Japan), Kentaro Kikuchi (Deprtment of Internal Medicine, Teikyo University Mizonokuchi Hospital, Kawasaki, Japan), Hirotoshi Ebinuma (Division of Gastroenterology and Hepatology, Department of Internal Medicine, Keio Graduate School of Medicine, Tokyo, Japan), Tatsuo Kanda, Osamu Yokosuga (Department of Medicine and Clinical Oncology, Graduate School of Medicine, Chiba University, Chiba, Japan), Takashi Himoto (Department of Medical Technology, Kagawa Prefectural University of Health Sciences, Kagawa, Japan),Toshio Senju, Masaru Harada (Third Department of Internal Medicine, University of Occupational and Environmental Health, Kitakyushu, Japan), Kazumoto Murata, Masashi Mizokami (The Research Center for Hepatitis and Immunology, National Center for Global Health and Medicine, Ichikawa, Japan), Ken Shirabe, Yoshihiko Maehara (Department of Surgery and Science, Kyushu University Graduate School of Medical Sciences, Fukuoka, Japan), Noriyo Yamashiki, Tomohiro Tanaka (Organ Transplantation Service, The University of Tokyo, Tokyo, Japan), Sumito Tamura, Yasuhiko Sugawara, Norihiro Kokudo (Hepatobiliary- pancreatic Surgery Division, Artificial Organ and Transplantation Division, Department of Surgery, Graduate School of Medicine, The University of Tokyo, Tokyo, Japan), Akira Mori, Michiyasu Yagi, Shinji Uemoto (Division of Hepato-Biliary- Pancreatic and Transplant Surgery, Department of Surgery, Graduate School of Medicine, Kyoto University, Kyoto, Japan), Hirohito Tsubouchi (Department of Digestive and Life-style related Disease, Kagoshima University Graduate School of Medical and Dental Science, Kagoshima, Japan). This work was supported by Grants-in-Aid for Scientific Research from the Japan Society for the Promotion of Science to MN (\#26293181, \#23591006), KY (\#26293180) and YA (\#25860576); by a Grant-in Aid for Clinical Research from the National Hospital Organization to MN; and by a Grant from the Research Program of Intractable Disease provided by the Ministry of Health, Labour and Welfare of Japan to MN. This work was supported in part by the Biobank Japan project funded by the Ministry of Education, Culture, Sports, Science and Technology of Japan. 
1 Mells, G. F., Floyd, J. A., Morley, K. I., Cordell, H. J., Franklin, C. S., Shin, S. Y. et al. Genome-wide association study identifies 12 new susceptibility loci for primary biliary cirrhosis. Nat. Genet. 43, 329-332 (2011)

2 Hirschfield, G. M., Xie, G., Lu, E., Sun, Y., Juran, B. D., Chellappa, V. et al. Association of primary biliary cirrhosis with variants in the CLEC16A, SOCS1, SPIB and SIAE immunomodulatory genes. Genes Immun. 13, 328-335 (2012).

3 Juran, B. D., Hirschfield, G. M., Invernizzi, P., Atkinson, E. J., Li, Y., Xie, G. et al. Immunochip analyses identify a novel risk locus for primary biliary cirrhosis at $13 q 14$, multiple independent associations at four established risk loci and epistasis between 1 p31 and 7q32 risk variants. Hum. Mol. Genet 21, 5209-5221 (2012).

4 Nakamura, M., Nishida, N., Kawashima, M., Aiba, Y., Tanaka, A., Yasunami, M. et al. Genome-wide association study identifies TNFSF15 and POU2AF1 as susceptibility loci for primary biliary cirrhosis in the Japanese population. Am. J. Hum. Genet. 91, 721-728 (2012)

5 Jostins, L., Ripke, S., Weersma, R. K., Duerr, R. H., McGovern, D. P., Hui, K. Y. et al. Host-microbe interactions have shaped the genetic architecture of inflammatory bowel disease. Nature 491, 119-124 (2012).

6 Hirano, A., Yamazaki, K., Umeno, J., Ashikawa, K., Aoki, M., Matsumoto, T. et al. Association study of 71 European Crohn's disease susceptibility loci in a Japanese population. Inflamm. Bowel Dis. 19, 526-533 (2013).

7 Yamazaki, K., Umeno, J., Takahashi, A., Hirano, A., Johnson, T. A., Kumasaka, N. et al. A genome-wide association study identifies 2 susceptibility loci for Crohn's disease in a Japanese population. Gastroenterology 144, 781-788 (2013).

8 Yamazaki, K., McGovern, D., Ragoussis, J., Paolucci, M., Butler, H., Jewell, D. et al. Single nucleotide polymorphisms in TNFSF15 confer susceptibility to Crohn's disease. Hum. Mol. Genet 14, 3499-3506 (2005).

9 Aiba, Y. \& Nakamura, M. The role of TL1A and DR3 in autoimmune and inflammatory diseases. Mediators Inflamm. 2013, 258164 (2013).

10 Parkes, M., Cortes, A., van Heel, D. A. \& Brown, M. A. Genetic insights into common pathways and complex relationships among immune-mediated diseases. Nat Rev Genet. 14, 661-673 (2013).

11 Nakamura, M., Yasunami, M., Kondo, H., Horie, H., Aiba, Y., Komori, A. et al. Analysis of HLA-DRB1 polymorphisms in Japanese patients with primary biliary cirrhosis (PBC): the HLA-DRB1polymorphism determines the relative risk of antinuclear antibodies for disease progression in PBC. Hepatol. Res 40, 494-504 (2010).

12 Umemura, T., Joshita, S., Ichijo, T., Yoshizawa, K., Katsuyama, Y., Tanaka, E. et al. Human leukocyte antigen class II molecules confer both susceptibility and progression in Japanese patients with primary biliary cirrhosis. Hepatology 55, 506-511 (2012).

13 Okada, Y., Yamazaki, K., Umeno, J., Takahashi, A., Kumasaka, N., Ashikawa, K. et al. HLA-CW*1202-B*5201-DRB1*1502 haplotype increases risk for ulcerative colitis but reduces risk for Crohn's disease. Gastroenterology 141, 864-871 e861-865 (2011).
14 Arimura, Y., Isshiki, H., Onodera, K., Nagaishi, K., Yamashita, K., Sonoda, T. et al. Characteristics of Japanese inflammatory bowel disease susceptibility loci. J. Gastroenterol. 49, 1217-1230 (2014).

15 Nishida, N., Mawatari, Y., Sageshima, M. \& Tokunaga, K. Highly parallel and short-acting amplification with locus-specific primers to detect single nucleotide polymorphisms by the DigiTag2 assay. PLoS One 7, e29967 (2012).

16 Ohnishi, Y., Tanaka, T., Ozaki, K., Yamada, R., Suzuki, H. \& Nakamura, Y. A highthroughput SNP typing system for genome-wide association studies. J. Hum. Genet. 46, 471-477 (2001)

17 Bamias, G., Martin, C. 3rd, Marini, M., Hoang, S., Mishina, M., Ross, W. G. et al. Expression, localization, and functional activity of TL1A, a novel Th1-polarizing cytokine in inflammatory bowel disease. J. Immunol. 171, 4868-4874 (2003).

18 Bamias, G., Kaltsa, G., Siakavellas, S. I., Gizis, M., Margantinis, G., Zampeli, E. et al. Differential expression of the TL1A/DcR3 system of TNF/TNFR-like proteins in large vs. small intestinal Crohn's disease. Dig. Liver Dis. 44, 30-36 (2012).

19 Aiba, Y., Harada, K., Komori, A., Ito, M., Shimoda, S., Nakamura, H. et al. Systemic and local expression levels of TNF-like ligand $1 \mathrm{~A}$ and its decoy receptor 3 are increased in primary biliary cirrhosis. Liver Int. 34, 679-688 (2014).

20 Kakuta, Y., Ueki, N., Kinouchi, Y., Negoro, K., Endo, K., Nomura, E. et al. TNFSF15 transcripts from risk haplotype for Crohn's disease are overexpressed in stimulated T cells. Hum. Mol. Genet 18, 1089-1098 (2009).

21 Hitomi, Y., Kawashima, M., Aiba, Y., Nishida, N., Matsuhashi, M., Okazaki, H. et al. Human primary biliary cirrhosis-susceptible allele of rs4979462 enhances TNFSF15 expression by binding NF-1. Hum Genet. (in press).

22 Hedl, M., Lahiri, A., Ning, K., Cho, J. H. \& Abraham, C. Pattern recognition receptor signaling in human dendritic cells is enhanced by ICOS ligand and modulated by the Crohn's disease ICOSLG risk allele. Immunity 40, 734-746 (2014).

23 Akiba, H., Takeda, K., Kojima, Y., Usui, Y., Harada, N., Yamazaki, T. et al. The role of ICOS in the CXCR5+ follicular B helper T cell maintenance in vivo. J. Immunol. 175, 2340-2348 (2005).

$24 \mathrm{Ma}, \mathrm{C} . \mathrm{S}$. \& Deenick, E. K. Human T follicular helper (Tfh) cells and disease. Immunol. Cell Biol. 92, 64-71 (2014).

25 Wang, L., Sun, Y., Zhang, Z., Jia, Y., Zou, Z., Ding, J. et al. CXCR5 CD4 T follicular helper cells participate in the pathogenesis of primary biliary cirrhosis. Hepatology 61, 627-638 (2015)

26 Wang, Z., Wang, Z., Diao, Y., Qian, X., Zhu, N. \& Dong, W. Circulating follicular helper $T$ cells in Crohn's disease (CD) and CD-associated colorectal cancer. Tumour Biol. 35, 9355-9359 (2014).

27 Nakae, S., Iwakura, Y., Suto, H. \& Galli, S. J. Phenotypic differences between Th1 and Th17 cells and negative regulation of Th1 cell differentiation by IL-17. J. Leukoc. Biol. 81, 1258-1268 (2007).

28 Nakamura, M. Analysis of disease-pathways by susceptilbity genes in primary biliary cirrhosis. Inflammation and Regeneration 34, 78-86 (2014). 